\title{
Discussion on: Foundation design for gravity retaining walls under earthquake
}

by Riccardo Conti, Giorgio Caputo and Giulia MB Viggiani

The discussers read the paper by Pender (2018) with great interest. The author addresses the important issue of computing the pseudostatic critical acceleration of gravity/cantilever retaining walls, corresponding to which a plastic mechanism is activated within the soil-structure system and the wall starts to move under the applied earthquake. In fact, the critical acceleration is the key ingredient for the seismic design of these structures, controlling both the maximum internal forces and the final displacement (Conti and Caputo, 2018; Conti et al., 2013).

By referring to dry cohesionless backfill and foundation soils, the author concluded that bearing capacity failure is the controlling mechanism when dealing with the seismic design of gravity/cantilever walls.

The scope of this discussion is twofold: (a) to extend the results presented by the author; (b) to provide additional comments on the seismic design of such structures.

\section{Sliding as opposed to bearing capacity failure mechanisms - the role of cohesion}

The discussers agree that the bearing capacity failure is likely to be the 'natural' failure mechanism for a wall on cohesionless soil. This is consistent with experimental data (Conti et al., 2015; Kloukinas et al., 2015; Koseki et al., 2003) and numerical results (Smith and Cubrinovski, 2011). Viggiani and Conti (2016) reported the results of a parametric theoretical study aimed at identifying the critical failure mechanism for gravity walls on different foundation soils. Figures 1 and 2 show the normalised critical acceleration of the wall, $k_{c}=\min \left(k_{y}\right.$, lid,$\left.k_{y, q l i m}\right)$, for gravity and cantilevered retaining walls, respectively, highlighting the role played by cohesion. Also, numerical and theoretical results for cantilever walls on fine-grained soils indicate that the sliding mechanism usually controls the dynamic behaviour of the wall in undrained conditions (Conti and Caputo, 2018).

\section{Seismic design of gravity/cantilever walls}

While the concept of an admissible wall displacement has been widely accepted within the performance-based seismic design philosophy, the possibility of admitting wall tilting, related to a temporary attainment of the bearing resistance, is still a controversial issue. Indeed, many provisions and codes of practice still recommend that the wall should be designed ensuring an adequate safety margin with respect to a bearing failure of the foundation and assuming the sliding mechanism as the critical one (Anderson et al., 2008; PIANC, 2000). The rationale behind it is twofold: (a) an excessive wall tilting could induce a sudden collapse of the wall by overturning; (b) no reliable procedures are available to accommodate a mixed sliding- rotational failure mode within the well-established Newmark's approach.

On the one hand, the discussers agree with the author that a rational seismic design of gravity/cantilever walls should contemplate the possible activation of both mechanisms, instead of excluding a priori the expected rotation. As a matter of fact, the temporary mobilisation of the soil shear resistance beneath the foundation would not lead to a fragile failure of the system, provided that an excessive wall tilting is prevented. This is a fundamental difference with respect to a pure overturning mechanism, which is indeed a fragile mechanism by nature.

On the other hand, further research is required to develop reliable (and simple) theoretical models, capable of handling combined tilting and sliding failure modes as, in this case, a direct application of the Newmark's sliding block procedure can lead to a significant under-prediction of the final displacement. 


\section{REFERENCES}

Anderson DG, Martin GR, Lam I and Wang JN (2008) Seismic Analysis and Design of Retaining Walls, Buried Structures, Slopes, and Embankments. Transportation Research Board, Washington, DC, USA, NCHRP Report 611.

Conti R and Caputo G (2018) A numerical and theoretical study on the seismic behaviour of yielding cantilever walls. Géotechnique, https://doi.org/10.1680/igeot.17.P.033.

Conti R, Viggiani GMB and Cavallo S (2013) A two-rigid block model for sliding gravity retaining walls. Soil Dynamics and Earthquake Engineering 55: 33-43.

Conti R, Madabhushi GSP, Mastronardi V and Viggiani GMB (2015) Centrifuge dynamic tests on gravity retaining walls: an insight into bearing vs sliding failure mechanisms. Proceedings of the 6th International Conference on Earthquake Geotechnical Engineering, Christchurch, New Zealand.

Kloukinas P, Scotto di Santolo A, Penna A et al. (2015) Investigation of seismic response of cantilever retaining walls: limit analysis vs shaking table testing. Soil Dynamics and Earthquake Engineering 77: 432-445.

Koseki J, Tatsuoka F, Watanabe K et al. (2003) Model tests of seismic stability of several types of soil retaining walls. In Reinforced Soil Engineering: Advances in Research and Practice (Ling HI, Leshchinsky D and Tatsuoka F (eds)). Marcel Dekker, New York, NY, USA, pp. 317-358.

Pender MJ (2018) Foundation design for gravity retaining walls under earthquake. Proceedings of the Institution of Civil Engineers - Geotechnical Engineering, https://doi.org/10.1680/igeen.17.00233.

PIANC (Permanent International Association of Navigation Congresses) (2000) Seismic Design Guidelines for Port Structures. Balkema, Rotterdam, the Netherlands.

Smith CC and Cubrinovski M (2011) Pseudo-static limit analysis by discontinuity layout optimisation: application of seismic analysis of retaining walls. Soil Dynamics and Earthquake Engineering 31(10): 1311-1323.

Viggiani GMB and Conti R (2016) On the behaviour of gravity retaining structures under seismic actions. Proceedings of the 1st International Conference on Natural Hazards and Infrastructure, Chania, Greece. 


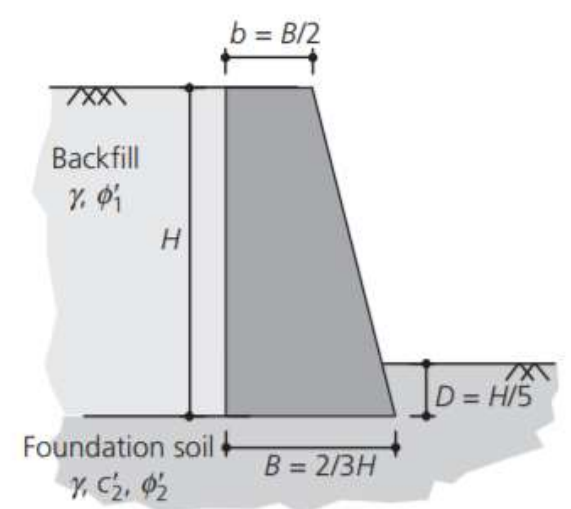

(a)

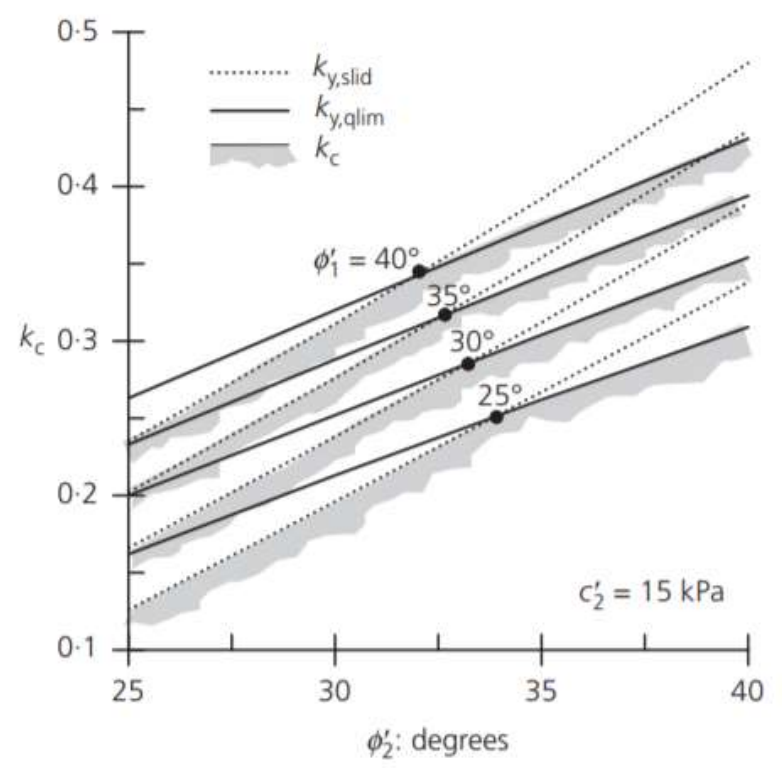

(b)

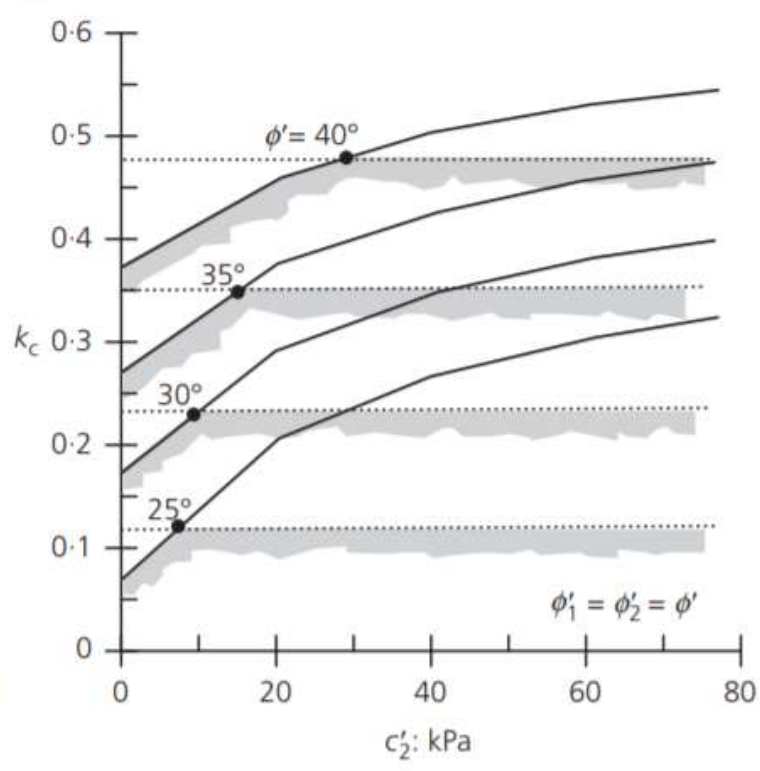

(c)

Figure 1. Gravity walls: (a) wall layout; (b) dependence of $k_{\mathrm{c}}$ on $\phi_{1}^{\prime}{ }_{1}$ and $\phi_{2}^{\prime}$, for $c_{2}^{\prime}=15 \mathrm{kPa}$; (c) dependence of $k_{\mathrm{c}}$ on $c_{2}^{\prime}$, for $\phi_{1}^{\prime}=\phi_{2}^{\prime}=\phi$ 


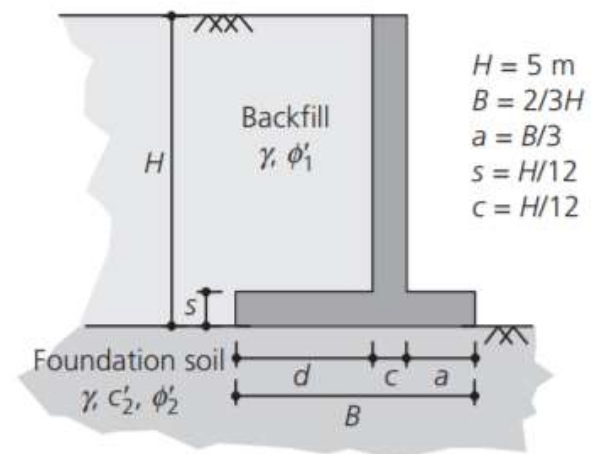

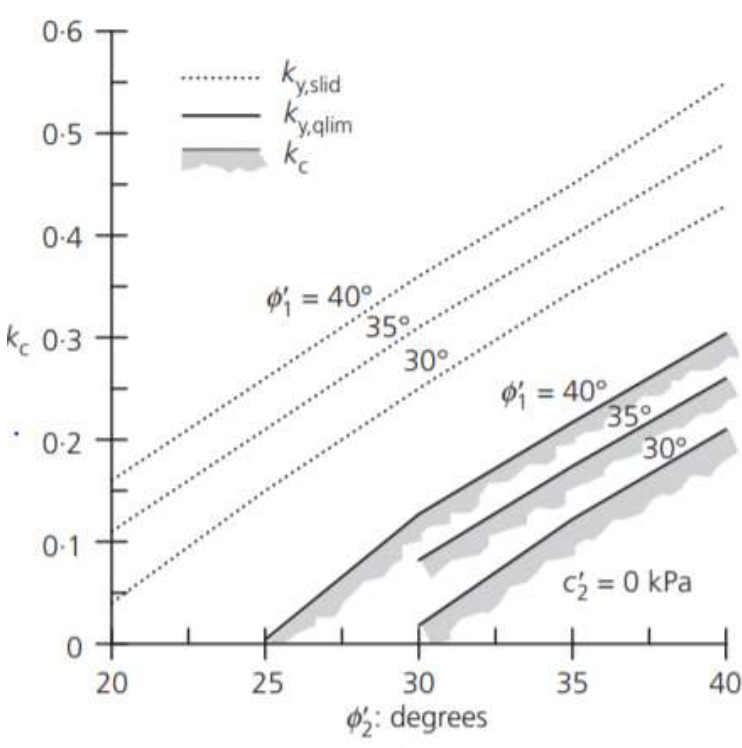

(b)

(a)

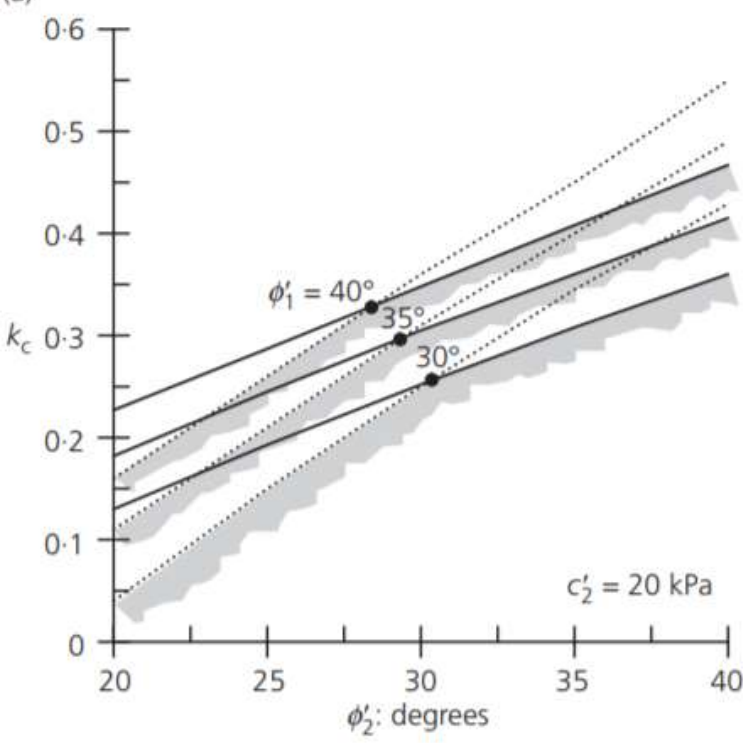

(c)

Figure 2. Cantilever walls: (a) wall layout; (b) dependence of $k_{c}$ on $\phi_{1}^{\prime}$ and $\phi_{2}^{\prime}{ }_{2}$, for $c^{\prime}{ }_{2}=0 \mathrm{kPa}$; (c) dependence of $k_{\mathrm{c}}$ on $\phi_{1}^{\prime}$ and $\phi_{2}^{\prime}$, for $c^{\prime}{ }_{2}=20 \mathrm{kPa}$ 